\title{
Archéopages
}

Archéopages

Archéologie et société

$46 \mid 2018$

Maisons

\section{Reconstitution et mise en chauffe d'un four à chaux}

S'interroger sur les découvertes en contexte archéologique

The reconstitution and heating of a lime kiln. Questioning discoveries in an archaeological context

Reconstrucción y calentamiento de un horno de cal. Interrogantes acerca de los descubrimientos en un contexto arqueológico

\section{Christophe Meloche}

\section{OpenEdition \\ Journals}

Édition électronique

URL : https://journals.openedition.org/archeopages/4510

DOI : 10.4000/archeopages. 4510

ISSN : 2269-9872

Éditeur

INRAP - Institut national de recherches archéologiques préventives

Édition imprimée

Date de publication : 1 décembre 2018

Pagination : $96-103$

ISSN : 1622-8545

Référence électronique

Christophe Meloche, "Reconstitution et mise en chauffe d'un four à chaux », Archéopages [En ligne], 46 | 2018, mis en ligne le 01 décembre 2020, consulté le 02 juin 2021. URL : http://

journals.openedition.org/archeopages/4510; DOI : https://doi.org/10.4000/archeopages.4510

(c) Inrap 


\title{
Reconstitution et mise
}

\section{en chauffe d'un four à chaux \\ S'interroger sur les découvertes en contexte archéologique}

\author{
Christophe Meloche ${ }^{\text {Irrap }}$
}

\section{6}

1. Fouille préventive au lieu-dit Corvée des Ruppes, sous la responsabilité de Yvan Virlogeux, Inrap. 2. Avec la participation de Philippe Delage Fabrice Maingot et Florian Ranucci, Guédelon.

3. Le substrat du site de Guédelon est totalement impropre à la fabrication de chaux.

4. La chaux utilisée à Guédelon est fournie par un chaufournier artisanal de l'Allier qui utilise un four à feu continu alimenté par des déchets d'hydrocarbure. Le chantier consomme une trentaine de tonnes de chaux par an.
La fouille en avril-mai 2014 d'un grand four à chaux, daté du début du XVI ${ }^{\mathrm{e}}$ siècle, à Châtenois dans le Jura (Virlogeux et al., à paraître), nous a conduit à proposer à l'équipe de Guédelon la construction d'un four à chaux. Cette expérience, qui a duré de juin à octobre $2015^{2}$, a apporté des informations intéressantes sur le fonctionnement de ce type de four, qu'il s'agisse du combustible, de la tenue du feu ou encore des matériaux calcaires qui permettent l'obtention de la chaux. Ces informations amènent ainsi à reconsidérer les structures découvertes en contexte archéologique.

\section{L'édification}

L'emplacement choisi pour la construction du four, au sud-est du site castral ${ }^{3}$, correspond au talus bordant l'ancienne carrière [ill. 1]. L'aire de chauffe a été établie sur un ressaut du terrain aplani tandis que la construction de la chambre de chauffe a nécessité d'entailler le talus. Le site du four à chaux domine le bassin de stockage de la chaux éteinte et l'aire de gâchage du mortier ${ }^{4}$. Le terrassement a débuté le 23 juillet avec quatre personnes et s'est achevé le lendemain, avec trois personnes. Le 5 août et le jour suivant, la chambre de chauffe a été délimitée et matérialisée au sol par la pose de plusieurs assises, l'emplacement du gueulard étant réservé. Ce travail a occupé une personne. La semaine du 31 août au 6 septembre a été consacrée à la construction de la chambre de chauffe et de sa voûte. Quatre personnes, dont deux maçons, ont pris en charge ce chantier ; la voûte en encorbellement a été achevée en deux jours. L'approvisionnement en pierres calcaires a occupé pendant chacun des jours de la semaine trois personnes sur une durée de 2 heures. Les moellons utilisés pour la chambre de chauffe provenaient d'une carrière située à Donzy (Nièvre), distante d'une trentaine de kilomètres. De nombreux blocs calcaires employés étaient issus des ateliers de taille du site de Guédelon. Pour constituer la charge à cuire, ces blocs ont été réduits à la masse, les plus petits morceaux étant disposés dans la partie haute du chargement. Dans la semaine du 7 au 11 septembre, a été maçonné devant la chambre de chauffe un mur de façade constitué de moellons ébauchés de grès ferrugineux (formation détritique de l'Albien), haut d'environ 2,20 m. Des murs de retour enserrent complètement la chambre de chauffe et vont s'adosser contre le talus. Les moellons ont été liés à la terre argileuse. Un maçon a été affecté à ce travail durant trois jours. La chambre de chauffe a un diamètre de $1,50 \mathrm{~m}$ et une hauteur maximale de $1 \mathrm{~m}$. La hauteur totale du four, depuis la plateforme accueillant la chambre de chauffe jusqu'au sommet de la charge à cuire, est de $3 \mathrm{~m}$. Au sol, les maçonneries en grès enserrant la chambre de chauffe forment un rectangle d'environ $4 \mathrm{~m}$ sur $2 \mathrm{~m}$.

Au cours de la semaine du 14 au 20 septembre, la charge à cuire - essentiellement des déchets de taille - a été disposée en tas de charge de section tronconique sur la voûte de la chambre de chauffe. Huit personnes ont été affectées à cette tâche. Le montage et le « cylindrage » ont occupé respectivement quatre et deux personnes, tandis que deux autres ont fendu du bois de chauffage constitué par des pieux en sapin de Douglas (provenant de la restitution ancienne de la palissade d'une motte castrale) et de branches de chênes. Le diamètre de tous ces bois variait entre 5 et $15 \mathrm{~cm}$. La semaine du 21 au 28 septembre a vu la 
fin du chargement de la charge à cuire. Une demijournée à deux personnes a été employée pour commencer à recouvrir la partie visible de la charge à cuire d'un manteau argileux. Le mur de façade a également été recouvert d'un torchis de terre tamisée mêlée à de la paille. La fente des bois de chauffage s'est poursuivie. Le bois a été stocké contre la halle abritant l'aire de gâchage. Ces deux tâches ont mobilisé deux personnes. Pendant la dernière semaine consacrée à la construction du four et au montage de la charge à cuire, deux personnes ont, pendant une demi-journée, achevé d'appliquer sur le dôme contenant la partie supérieure du chargement une couche de chaux que surmontera le manteau argileux. Une autre demi-journée a été nécessaire à l'achèvement de l'enduit du mur de façade par trois personnes. Une demi-journée a été consacrée à la construction d'une porte amovible pour le four, constituée d'un cadre et d'un clayonnage en osier ourdis de torchis. Cette porte ne supporta pas la chaleur et fut très vite détruite par le feu dès lors qu'elle était en position d'obstruction du gueulard. La porte fermant le gueulard d'un des fours du $\mathrm{Xv}^{\mathrm{e}}$ siècle étudié par Valérie Viscusi à Besançon au travers de la comptabilité était en métal (Viscusi, 2006). Le gueulard de l'alandier d'un four daté du $\mathrm{III}^{\mathrm{e}}$ siècle de notre ère fouillé à Besançon pouvait être fermé par une grosse dalle en grès (Munier, 2006).

Au total la masse de calcaire, charge à cuire et construction de la voûte ont nécessité l'emploi d'environ neuf tonnes de calcaire. Le volume de matériaux employés est estimé par le maçon à $4,5 \mathrm{~m}^{3}$, soit une masse de 2 tonnes par $\mathrm{m}^{3}$.

\section{La cuisson}

La cuisson a commencé le 19 octobre à 10 h 50 et s'est achevée le 23 octobre à 20 h. Elle a été conduite par deux à trois personnes, l'une surveillant le foyer, et les autres s'occupant de l'approvisionnement en bois, stocké hors de l'aire de chauffe.

Le feu a été allumé à l'aide de petits bois au milieu de la chambre de chauffe [ill. 2]. D'importantes fumées blanchâtres (vapeur d'eau) se sont échappées par la partie du four non recouverte d'un manteau d'argile. Deux personnes ont alors enduit les moellons pour que tous les interstices soient obturés. Le feu a été alimenté en bois d'abord tous les trois-quarts d'heure puis tous les quarts d'heure. Au bout de quatre heures, ni le four ni l'encaissant n'étaient secs et le travail consistant à compléter le manteau d'argile a dû être repris au cours de l'après-midi. À 18 h, des fumeroles bleutées sont apparues à nouveau le long du pignon de la chambre de chauffe, passant par des petites fissures du manteau. Le travail s'est poursuivi la nuit, effectué par deux chaufourniers de Guédelon. Au cours de la première partie de la nuit, il est devenu difficile de laisser la main sur les parements extérieurs du fait de l'élévation importante de leur température. L'équipe de nuit a alimenté le feu exclusivement avec du chêne. Au total, six stères de bois ont été brûlés dont deux durant la nuit.

Le 20 octobre à $9 \mathrm{~h}$, le four et l'encaissant avaient séché, l'environnement de vapeur d'eau n'était plus prégnant. On a pratiqué dans la coupole un évent supplémentaire mais, le tirage demeurant difficile, deux autres évents ont dû être percés à la base du dôme. Malgré cela, on a constaté qu'un excédent de braises engorgeait la chambre de chauffe et que le brasier ne produisait que très peu de flammes. Une partie de la braise a été évacuée. Deux autres évents ont été percés et le feu conduit à l'entrée du gueulard et non dans la chambre de chauffe. Un vent léger et l'appel d'air en provenance de la chambre de chauffe ont permis aux flammes, vives désormais, d'entrer largement dans la chambre de chauffe. On a retiré par deux fois de la braise hors de la chambre de chauffe. À midi, l'alimentation en bois du feu, toujours conduit depuis le gueulard, a été augmentée. Au bout d'une heure, on a retiré encore, par deux fois, de la braise de la chambre de chauffe. Ce n'est qu'à 14 h 50 que l'on a constaté la diminution des émanations de vapeur d'eau sous forme de fumées blanchâtres et l'intensification de la chaleur. À partir de 16 h 5o, le feu a été conduit au-delà du gueulard et le foyer rapproché du centre de la chambre de chauffe. Il s'est alors dégagé une odeur légèrement âcre qui a perduré jusqu'à la fin de la cuisson.

Le 21 octobre, l'équipe de nuit a noté l'apparition, à $4 \mathrm{~h} 05$, de lueurs rouge vif aux évents, suivie à $6 \mathrm{~h} 50$ de flammes courtes s'échappant par les évents percés au nord dans le sommet de la chambre de chauffe. Trois stères ont été brûlés durant la nuit et, à $9 \mathrm{~h} 20$, la consommation de bois, depuis le 20 octobre au matin, a été estimée à neuf stères. Des fentes dans la maçonnerie extérieure de la chambre de chauffe sont apparues sans remettre en cause la stabilité de l'ensemble. On a colmaté les fissures à l'argile. À $10 \mathrm{~h}$, l'enlèvement d'une partie des braises de la chambre de chauffe a provoqué un rejet abondant par les évents de fumées noires et grasses. Ce n'est qu'à 11 h qu'il a enfin été possible de conduire le feu au cœur de la chambre de chauffe, ce qui n'était plus arrivé depuis plus de 24 heures. L'alimentation en bois a ensuite été augmentée très nettement. À partir de ce jour, les moellons composant la chambre de chauffe ont régulièrement changé d'aspect, devenant blanc crémeux, puis blanc lumineux avec des effets rose pâle.

La quantité de bois utilisé n'a été mesurée que jusqu'au troisième jour : elle atteignait alors 16 stères. On peut estimer qu'il faut rajouter au minimum 6 stères pour les dernières 24 heures, soit un total de 22 stères. Rappelons que le cubage de bois à brûler est estimé par les archéologues suisses entre 1,5 et 3 stères par cubage de pierre (Demarez, 2014). Si l'on retient la fourchette basse de cette estimation, les $16 \mathrm{~m}^{3}$ du volume de la 
1. a. Creusement du talus à

l'emplacement du futur four.

b. Vue générale du

chargement de la charge

à cuire au-dessus de

la chambre de chauffe. c. Chargement de la charge

à cuire sur l'extrados

de la voûte de la chambre

de chauffe.

d. Vue latérale du fronton

de grès. a

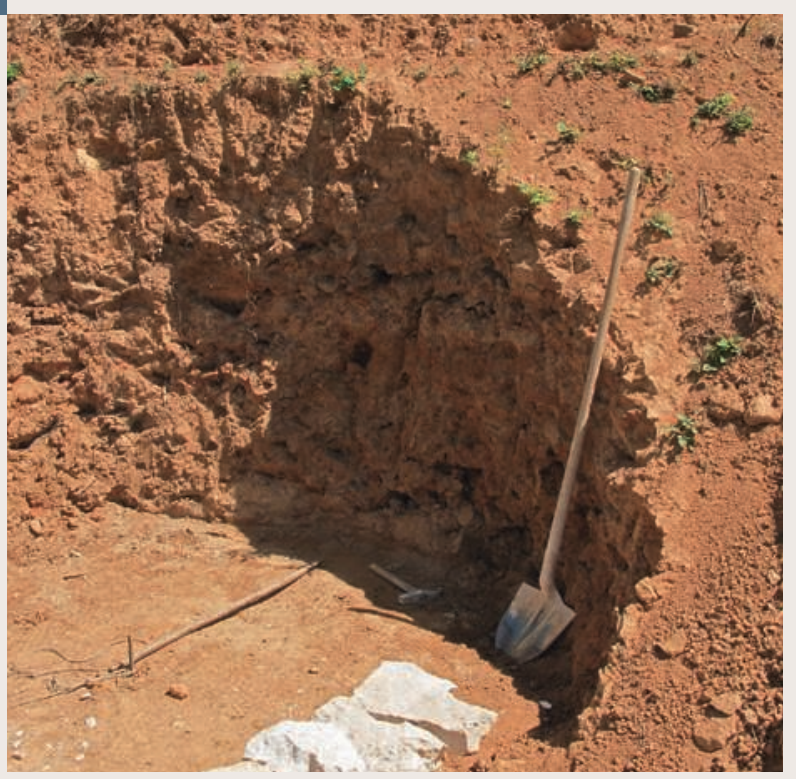

c

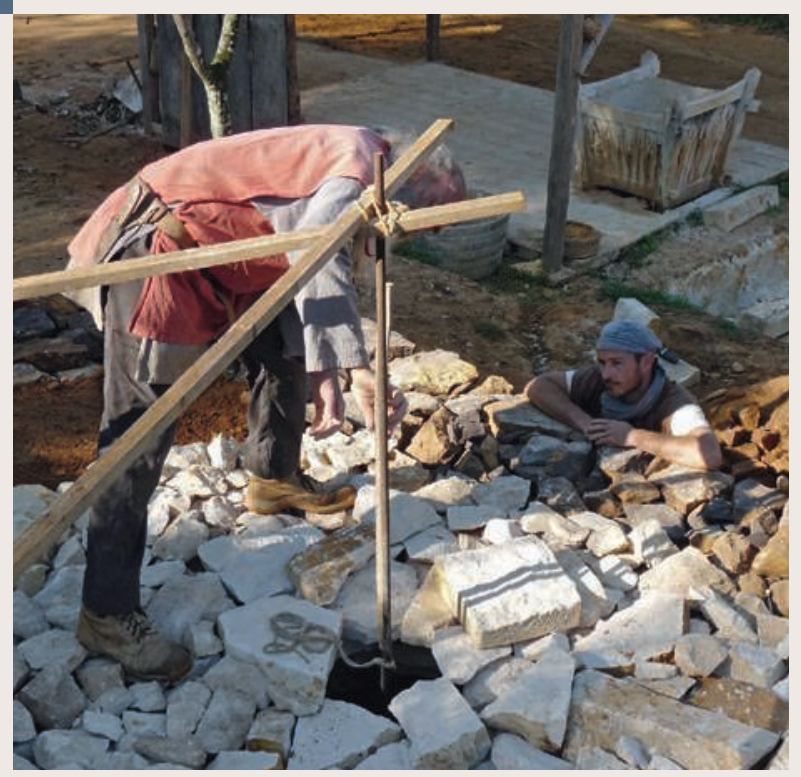

b

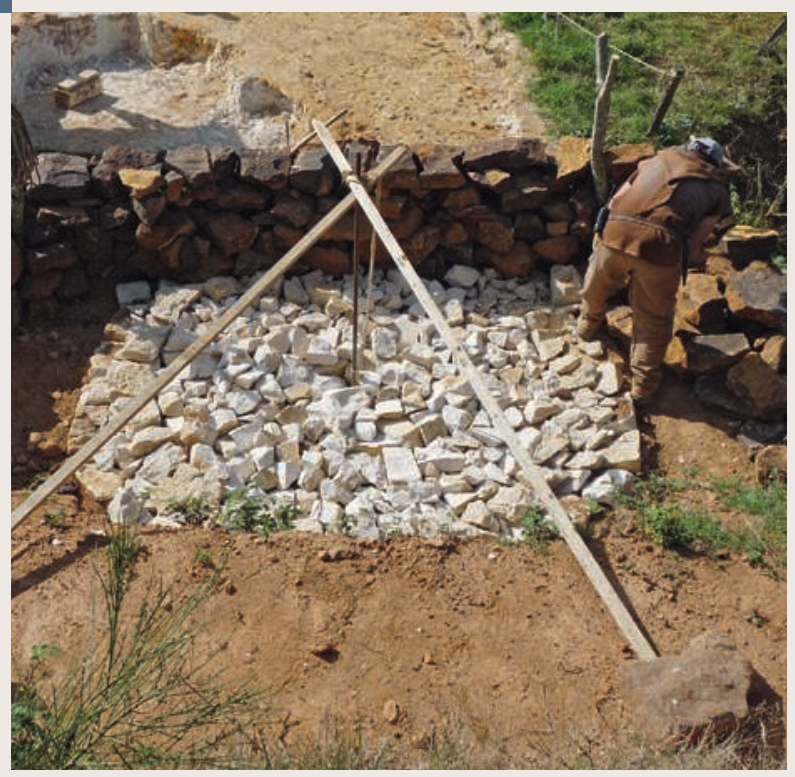

d

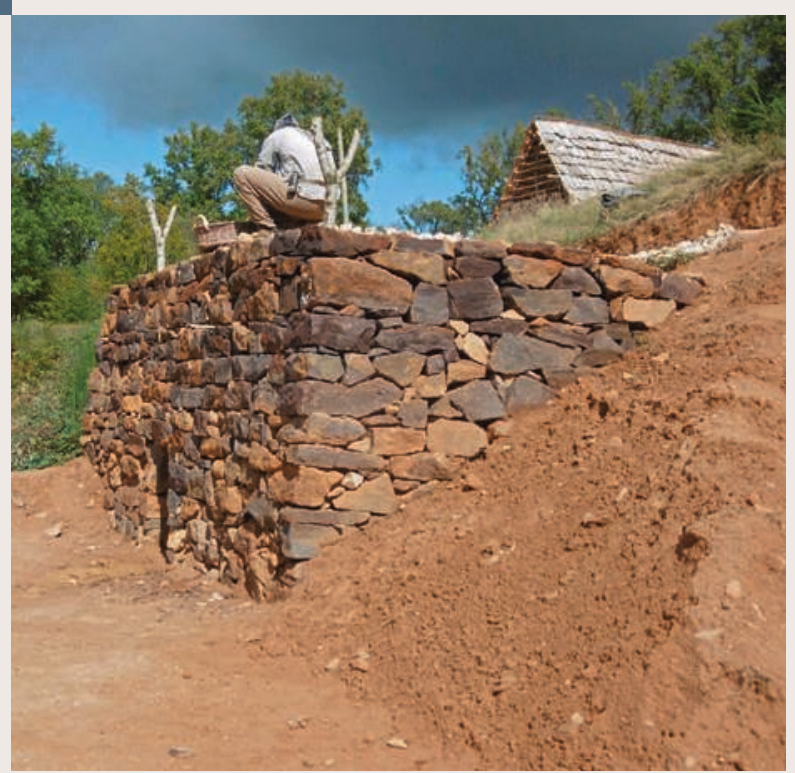


e

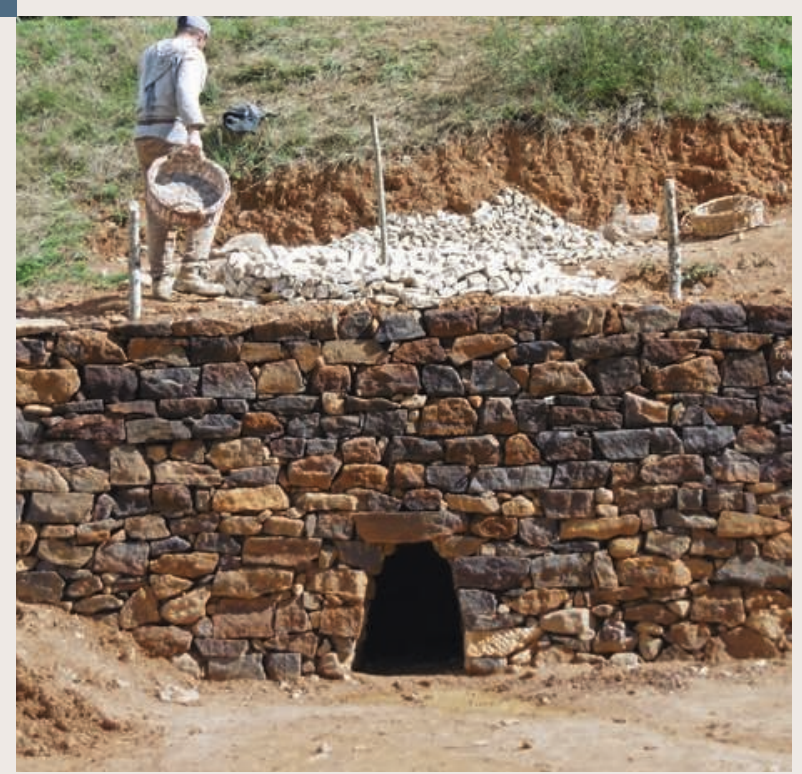

g

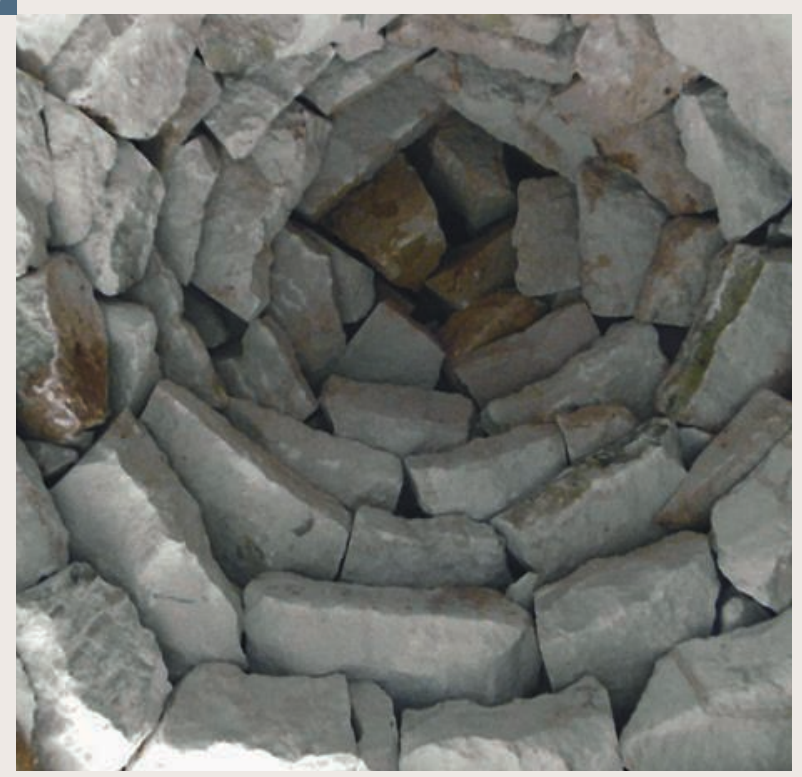

f

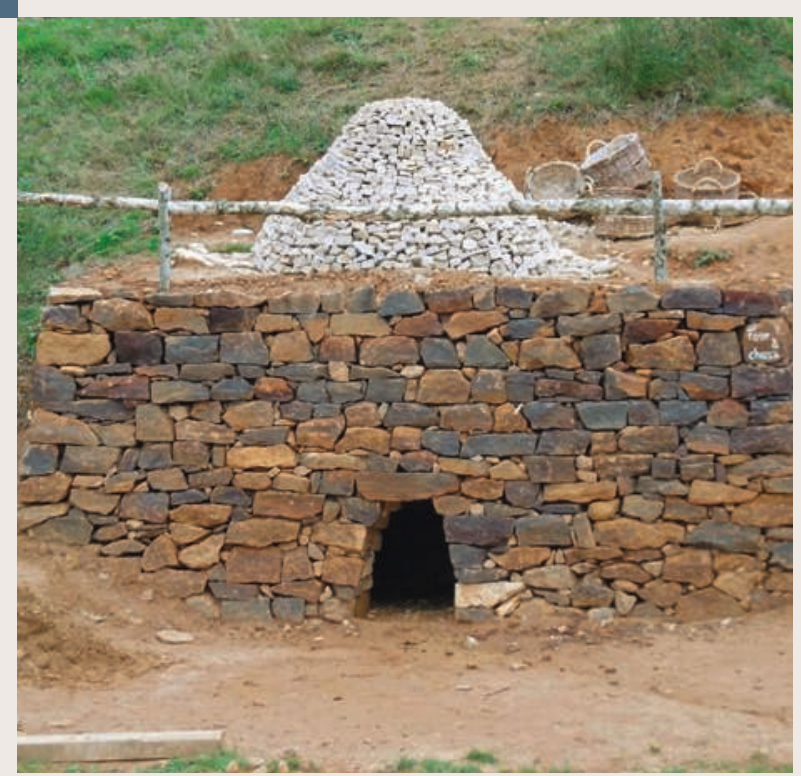

h

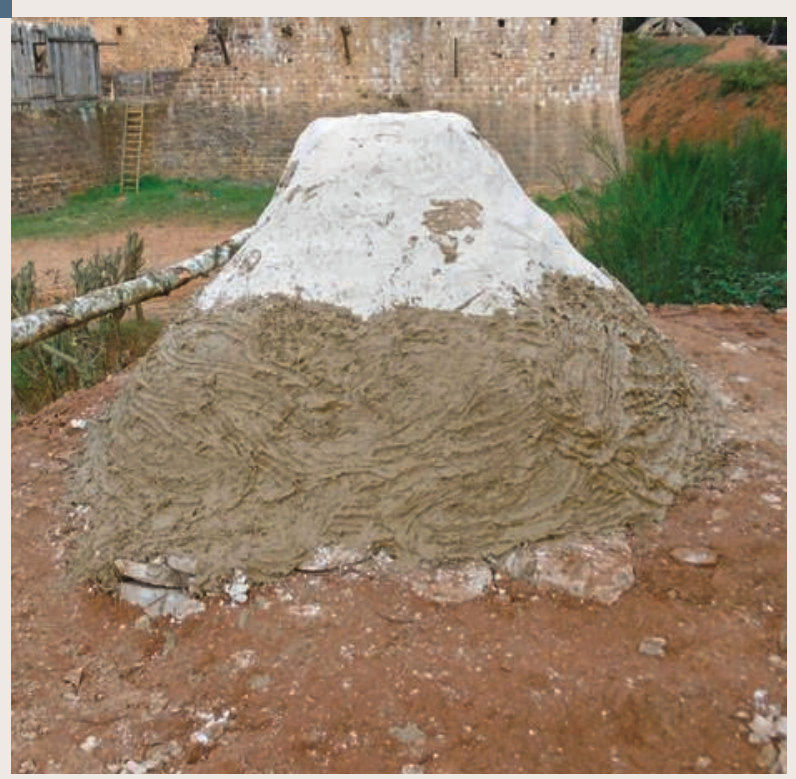

?)

$\stackrel{\infty}{\stackrel{\infty}{\sim}}$

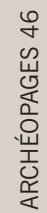


2. a. Petit bois positionné au

centre de la chambre de

chauffe avant l'allumage.

b. Émissions de vapeur d'eau

au cours des premières

heures de cuisson. c. Feu conduit dans l'aire de

chauffe depuis le gueulard.

d. Manteau recouvrant le

dôme pendant la cuisson. a

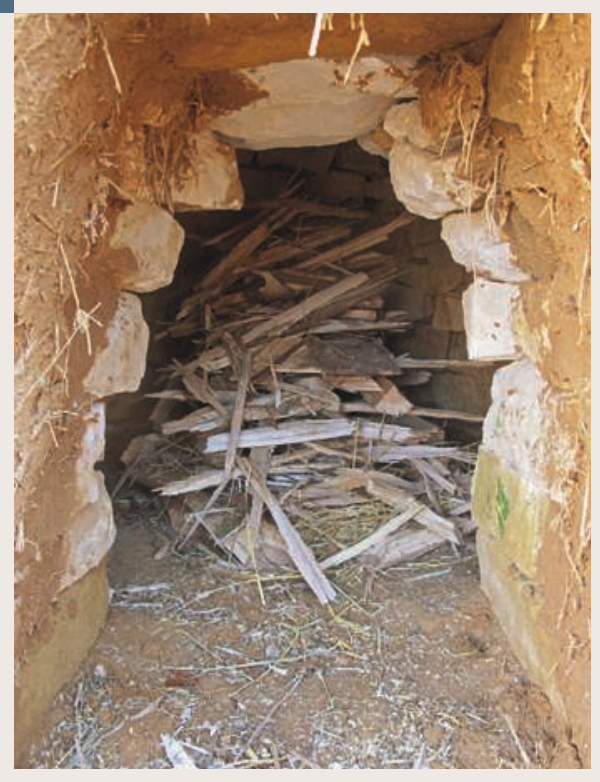

b

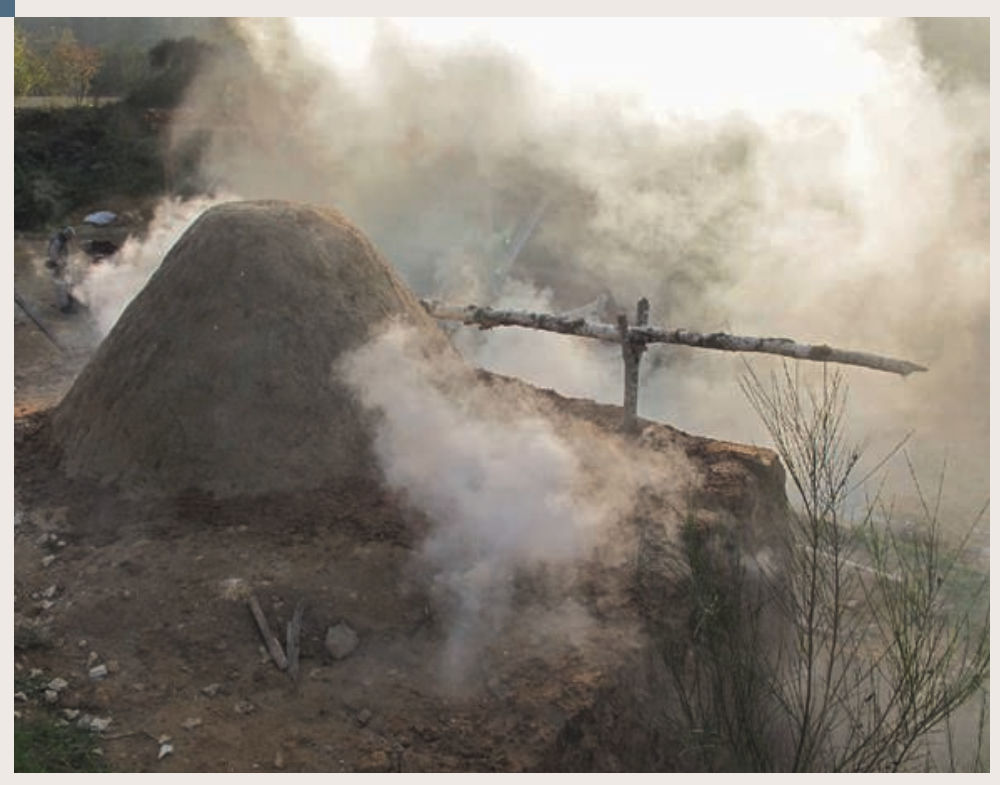

c

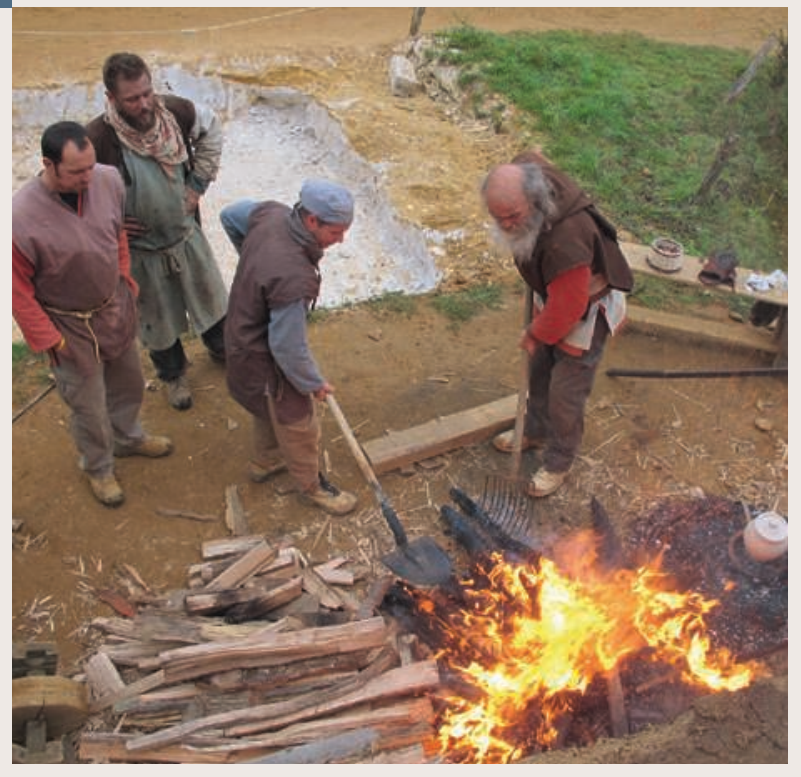

d

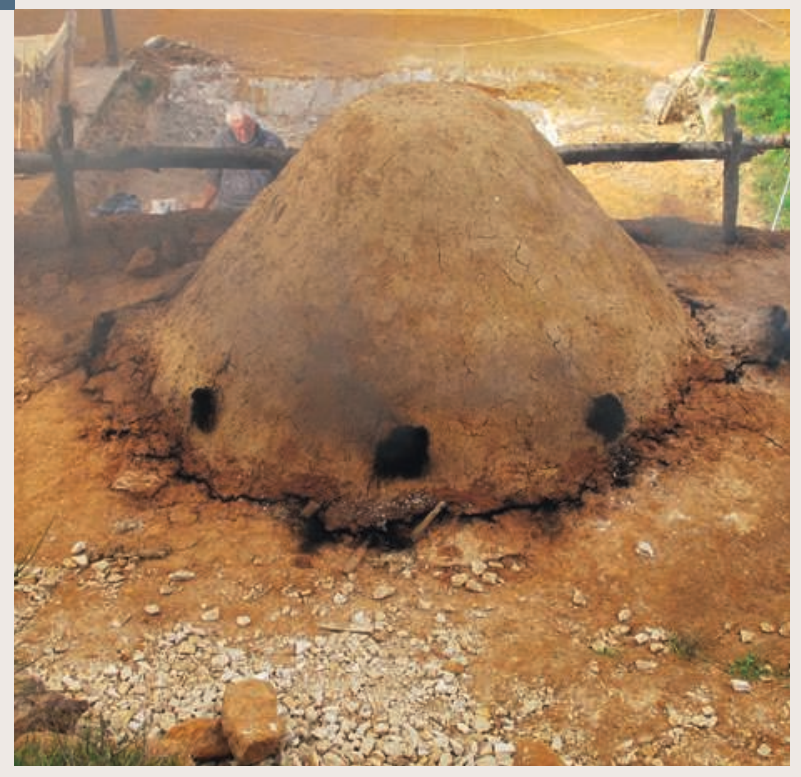


e

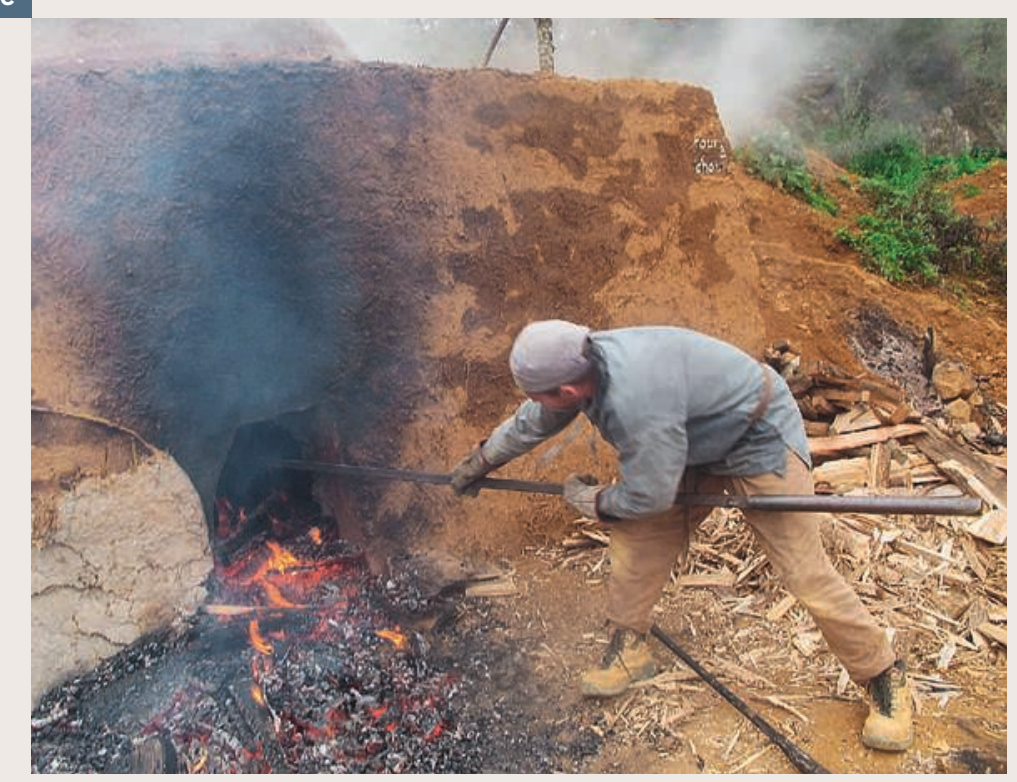

f

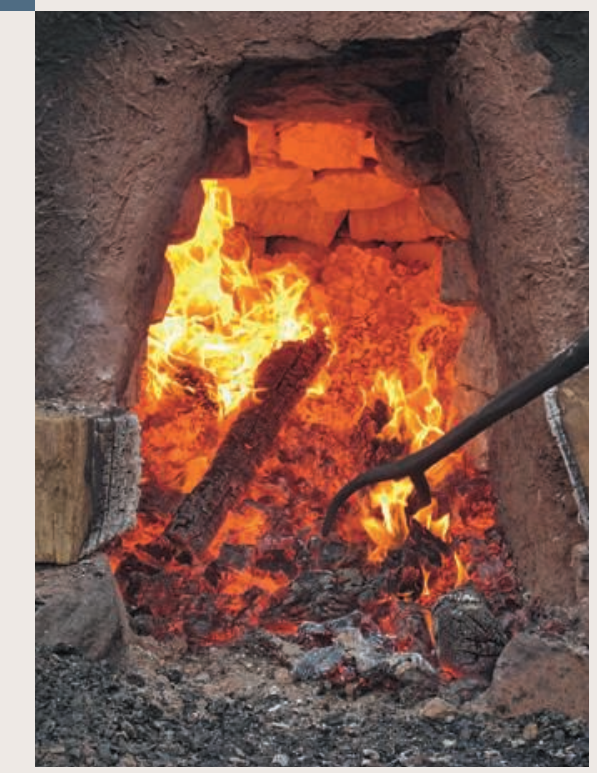

g

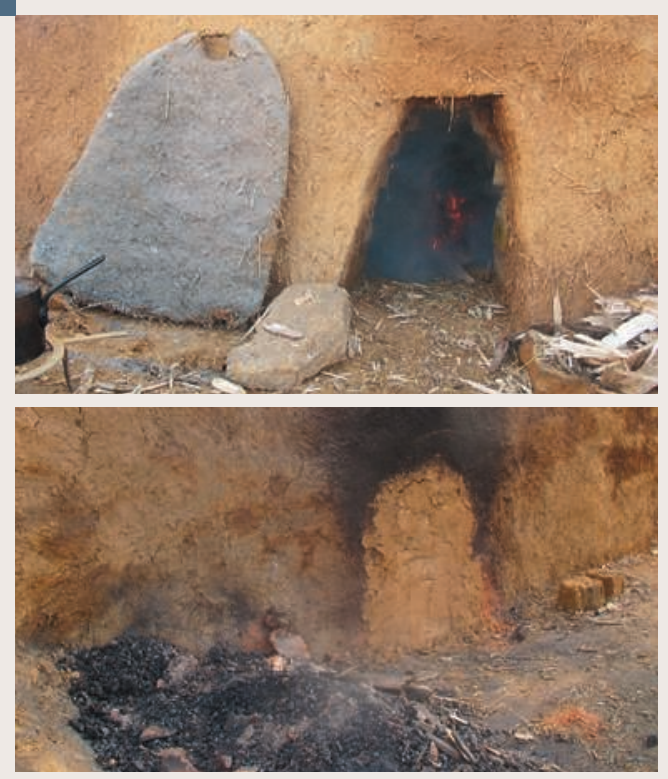

h

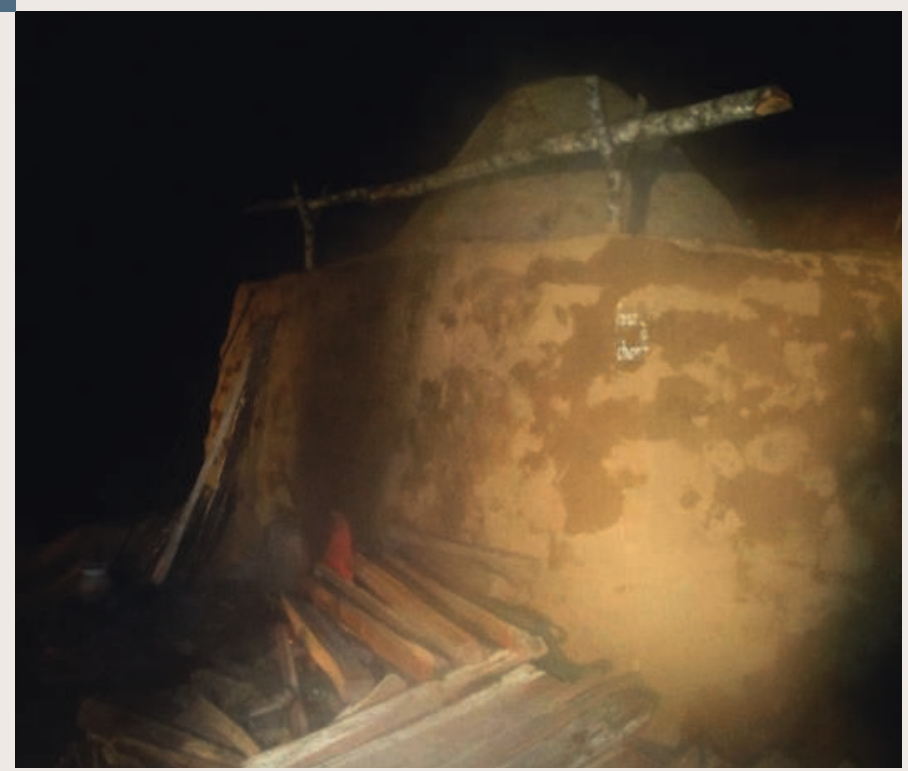

훙 
3.Vue générale des blocs de chaux vive obtenus après quatre jours de cuisson, au niveau de la chambre de chauffe, après démontage de sa voûte.
4. Extinction de la chaux vive en plongeant les blocs dans l'eau : la couleur laiteuse de l'eau est due aux particules de chaux.
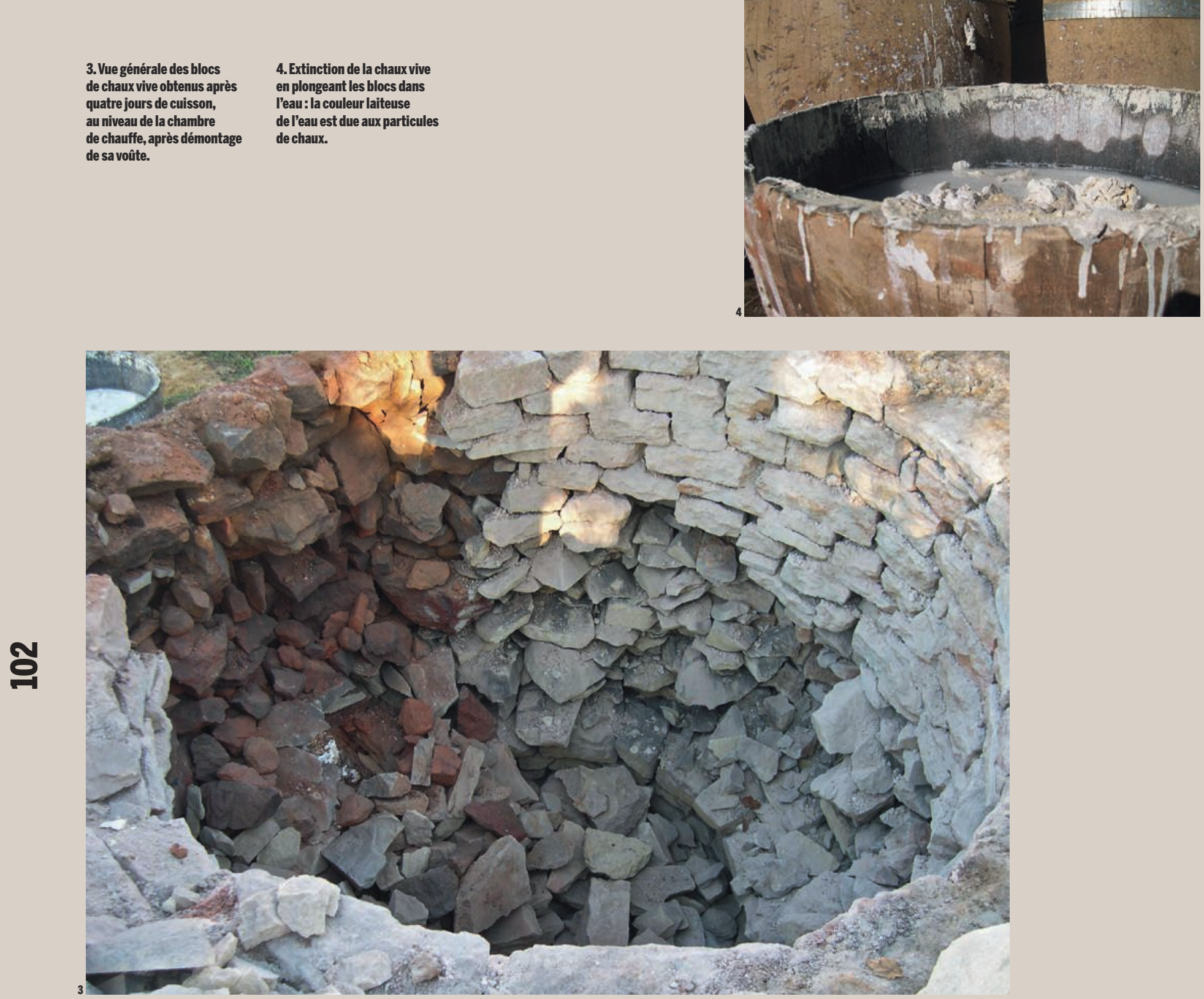

Du calcaire à la chaux

En se calcinant, le calcaire (carbonate de calcium- $\mathrm{CaCO}_{3}$ ) perd son gaz carbonique et se transforme en chaux vive (oxyde de calcium-CaO). Le dégagement de dioxyde de carbone $\left(\mathrm{CO}_{2}\right)$ est important lors de la calcination et, par voie de conséquence, la perte de la masse volumique de la charge à cuire peut varier entre 15 et $45 \%$. Jean-Pierre Adam rappelait que le combustible employé pour les fours à « longue flamme » devait être de « petites dimensions, bien sec et laisser échapper rapidement ses gaz inflammables "

(Adam, 1989, p. 71) ; les fours à longue flamme sont des fours avec foyer à la base pouvant être alimenté en combustible en cours de cuisson, à la différence des fours par empilement dont la chambre de chauffe est remplie de combustibles et de charge à cuire, et de la cuisson en aire ouverte. Le même auteur mentionne que dans des fours du premier type (dont le volume variait de 25 à $120 \mathrm{~m}^{3}$ ) cuisant artisanalement du calcaire à la fin du siècle dernier, en Grèce, en Tunisie et en Italie, la nature des combustibles variait : noyaux d'olives et coques d'amandes, herbes sèches, menus branchages provenant de buissons, pommes de pins...

Une cuisson pouvait durer plusieurs jours, voire une semaine en fonction du volume de la charge. Une température stable de $1000^{\circ}$ environ doit être maintenue dans le four (Othenin-Girard, 2007). Il en résulte des blocs de couleur laiteuse que l'on nomme chaux vive. On éteignait la chaux vive en ajoutant de l'eau sur les pierres ainsi transformées. Cette hydratation accélérait la désintégration des blocs en produisant un fort dégagement de chaleur. On obtient un hydroxyde de calcium $\left(\mathrm{Ca}(\mathrm{OH})_{2}\right)$ ou chaux aérienne en ajoutant des volumes d'eau non quantifiés. Cette extinction de la chaux se faisait sur les sites de consommation, c'est-à-dire sur les chantiers de construction. Si la charge calcaire à cuire contient entre $8 \%$ et $20 \%$ d'argile, on obtient une chaux dite hydraulique dont la propriété essentielle consiste en ce que « la prise peut s'effectuer en milieux aqueux [....] toutefois, au-delà de $20 \%$ d'argile, les calcaires ne sont plus utilisables pour la chaux... " (Ibid.). 
charge de Guédelon devaient nécessiter de brûler 24. stères, ce qui correspond bien aux 22 stères consommés lors de cette expérience.

Le démontage du four et l'extinction de la chaux

Cinq jours ont été nécessaires pour éteindre le four. L'équipe Inrap n'ayant pas été présente pendant cette période, les observations suivantes sont issues des notes prises par Fabrice Maingot à Guédelon. Lorsque la cuisson s'est achevée le 23 octobre, les maçonneries étaient très chaudes. On a procédé, le 26 octobre, au démontage du dôme constitué de pierres marbrières [ill. 3]. La chaleur était encore trop intense pour poursuivre. Ce n'est que le 29 octobre que l'on a pu atteindre la pierre calcaire mi-dure. Toutes les pierres du dôme n'étaient pas cuites : seules les plus légères, situées au niveau des évents et en dessous, l'étaient suffisamment pour être transformées en chaux. Après un minimum de 30 minutes d'immersion dans l'eau, les pierres bien cuites commencent par se désagréger sous l'effet de la chaleur produite par la réaction chimique (une fumée se dégage), puis elles se transforment en pâte [ill. 4]. Il faut cependant mélanger à plusieurs reprises et ajouter de l'eau le temps que la chaleur s'estompe. Au terme de l'expérience, nous avons rempli sept tonneaux de chaux (environ 1,5 tonne).

Il serait prétentieux de faire un retour d'expérience exhaustif non seulement parce que les agents de l'Inrap n'ont pu rester pendant toute la durée de l'expérience mais encore parce qu'ils ne possédaient ni les compétences techniques, ni les instruments de mesure qui auraient permis d'apprécier le temps de montée en chauffe et les degrés obtenus. Notons à ce propos que cette expérience était une première à Guédelon et qu'aucun chaufournier professionnel n'a été présent à une quelconque étape de cette réalisation. C'était donc une expérience totalement empirique : tout ce que nous savions, les uns comme les autres, venait de lectures d'ouvrages spécialisés ou archéologiques, et de quelques rares échanges, pour les gens de Guédelon, avec des chaufourniers professionnels espagnols travaillant « à l'ancienne ». Ainsi nul ne savait quand le processus de la réaction chimique de la décarbonatation du calcaire s'accomplissait. Était-ce à partir du changement d'aspect du calcaire observé le matin du troisième jour ? Mais il convient de rappeler que l'équipe de Guédelon réalise depuis un certain temps avec succès la cuisson de tuiles, briques, carreaux de terre cuite confectionnés sur place. Elle a acquis un savoirfaire certain dans la conduite de ces cuissons. Le montage parfait du four par les maçons de Guédelon, possédant une excellente maîtrise des maçonneries en pierre sèche, a permis à la structure de chauffe de ne pas s'effondrer lors de son utilisation. Comme l'a montré Valérie Viscusi, la construction de grands fours à chaux commandités par la commune de Besançon au $\mathrm{XV}^{\mathrm{e}}$ siècle requérait la présence de maçons et de charpentiers (Viscusi, 2006). Il est évident que, pour des fours en milieu rural comme celui de Châtenois, tous ces corps de métiers n'étaient pas présents. Si le four construit à Guédelon n'était pas une reproduction du modèle fouillé à Châtenois, il a été édifié avec la même méthodologie que celle employée jusqu'à l'époque industrielle. Il en aura été de même pour son fonctionnement et son défournement.

Cette expérience fut une réussite puisque l'on a pu produire de la chaux en quantité non négligeable. Cependant, et cela est riche d'enseignements, eu égard à la masse de calcaire à brûler, les quantités de chaux obtenues furent moins importantes que l'on s'y était attendu. La raison en réside d'abord dans le matériau utilisé : le calcaire dit « marbrier » par les maçons de Guédelon s'est révélé trop riche en silice. De plus, ce matériau se trouvait placé dans la partie haute du dôme et donc la plus difficile à brûler. Il apparaît en effet que la charge qui a subi la transformation chimique adéquate était constituée essentiellement par les moellons rentrant dans la composition même de la chambre de chauffe. Notre second constat est que la conduite du feu en employant par moments uniquement du chêne fut contreproductive. Les différentes observations faites à divers stades de cette opération nous ont permis d'envisager la fouille des fours à chaux de façon très sensiblement différente et de reconsidérer l'interprétation de certains faits observés dans ces structures de combustion.
Références bibliographiques

ADAM J.-P., 1989, La construction romaine : matériaux et techniques, Paris, Picard, 367 p.

Demarez J.-D., 2014, La production de chaux en Ajoie (Jura suisse) de l'époque romaine au XIXe siècle. Recherches d'archéologie et d'histoire, Porrentruy, Office de la culture et Société jurassienne d'émulation, $151 \mathrm{p}$.

Munier C., 2006, Besançon (Doubs), îlot Pasteur, rapport d'opération, Inrap-SRA Bourgogne, $98 \mathrm{p}$.

OTHENIN-GiRARD B., 2007, « Les fours à chaux de Boncourt, Grands'Combes, (Jura CH) : époque romaine, haut Moyen Âge, $\mathrm{XVIII}^{\mathrm{e}}$ siècle », in BÉLETGonda C., Mazimann J.-P., Richard A. et al. (dir.), Mandeure, sa campagne et ses relations d'Avenches à Luxeuil et d'Augst à Besançon, Actualités archéologiques régionales, Besançon, Presses universitaires de Franche-Comté, p. 229-237.

VIscusi V., 2006, Besançon Palais de Justice, Four à chaux, les données d'archives, ACR Besançon, inédit, $6 \mathrm{p}$.

Virlogeux Y., Burgevin A., Fort B., Joan L., Lamy V., Meloche C., à paraître, Châtenois, Corvée des Ruppes, Le Prunier, Rochefort-sur-Nenon, Mont-Ayer, Au Carré, Lazard (Jura), étude d'un tronçon de la voie antique Dijon-Salins-les-Bains et d'un four à chaux du XVI siècle, rapport d'opération, Inrap-SRA Bourgogne-Franche-Comté, à paraitre. 